Aus dem Pharmakologischen Institut der Universität in Wien.

\title{
Ueber nicht zündende Subkutaninjektion entzündlich wirkender Heilmittel.
}

Von Prof. H. H. Meyer and Dr. P. Freund.

Manche Heilmittel sind vom Verdauungskanal aus ganz unwirksam und müssen unter allen Umständen parenteral beigebracht werden. Andere werden zwar vom Darmkanal ganz oder teilweise unverändert resorbiert und können ihre Allgemeinwirkung ausïben; doch hängt Beginn, Verlauf und Grad der Wirkung wesentlich ab von den jeweiligen Resorptionsbedingungen und von dem Durchgang des resorbierten Stoffes durch die Leber. Ist die Resorption wegen Mageninsuffizienz oder Stauung in der Porta und Leber behindert, so kann die gewünschte Wirkung ungenügend sein oder ganz ausbleiben. Vomi Rektum aus gelangen die im Einlaut beigebrachten Stoffe allerdings teilweise durch die Hämorrhoidalvenen unmittelbar. in den großen Kreislauf und so zu verhältnismäßig. sicherer und rascher Wirkung. Am sichersten aber wird dies durch parente rale Zufuhr erreicht, also durch subkutane, intramuskuläre oder intravenöse Injektion. Die in travenöse Einspritzung kann nur von geübter Hand an ruhig haltenden 'Kranken richtig ausgeführt werden und setzt auBerdem das Auffinden einer geeigneten Vene für die perkutane Injektion voraus. Wo diese Bedingungen, wie bei Kindern, sehr fettreichen Kranken u. a., nicht erfüllbar sind, ist man zur intramuskulären oder subkutanen Injektion genötigt. Dieser Art der Zufuhr steht bei nicht wenigen Arzneistoffen der Uebelstand hindernd entgegen, daß sie örtlich mehr oder weniger stark reizen und an der Injektionsstelle aseptische Entzündung, Schmerz und Schwellung bis zu heftigster Phlegmone hervorrufen. Das gilt gerade von einigen sehr viel gebrauchten Mitteln, u. a. namentlich den digitalisartig wirkenden Glukosiden und Glukosidgemischen sowie vom Neosalvarsan.

Es schien uns daher der Mühe wert, zu versuchen, die örtlich entzündungserregende Wirkung solcher Arzneimittel zu beseitigen. Das gelingt nun in der Tat unter Zuhilfenahme lok alan äs thesierender Stoffe, wie Novokain, Alypin, Stovain u. a.

Die wichtige Erfahrung, daß Lokalanästhesie eine örtlich bestehende Entzündung löschen oder wenigstens eindämmen kann, stammt bekanntlich von SpieB(1). Seine klinischen Beobachtungen hat der eine von uns vor längeren Jahren durch Herrn A N. Bruce (2) einer experimentellen Analyse unterziehen lassen und dabei feststellen können, daß die Entzündung unter dem Einfluß eines Reizstoffes, wie z. B. des Senföls, nicht zustandekommt, solange die sensiblen Nervenendigungen und vermutlich zugleich mit ihnen die vasodilatatorischen Nervenendapparate in den kleinen Gefäßen des betroffenen Gewebes funktionsunfähig sind - sei es dauernd durch Degeneration, sei es vorübergehend durch örtliche Betäubung. Diese letztere Möglichkeit, eine Reizentzündung von vornherein zu verhüten, haben wir nun auch bei den erwähnten Arzneimitteln geprüft und am Tier und Menschen entsprechende Versuche angestellt. Sie haben zu sehr günstigen Ergebnissen geführt, über welche im Folgenden berichtet wird.

Strophanthin, Digitoxin, Cymarin, Scillaren und Di. galen rufen subkutan eingespritzt keine Entzündung hervor, wenn jeder Dosis eine entsprechende Menge Novokain, Alypinoder Stovain zugesetzt is t. Experimente in der gleichen Richtung mit Neosalva rsan - intramuskulär injiziert - ergaben; wenn dieser sehr labile Stoff durch ein besonderes Verfahren zuerst gegen Ausfällung und Zersetzung durch das Anästhetikum geschützt ist, ähnliche Resultate; doch sind darüber weitere Versuche noch im Gange, über die später berichtet werden soll.

A. Tierversuche: Eine erste Versuchsreihe hatte zu entscheiden, ob die genannten Heilmittel alle in gegebe $n$ beim Tier zuverlässig Entzündung hervorrufen. Das war bei Mäusen, Ratten und Meerschweinchen nicht der Fall; bei Kaninchen, an denen bereits $\mathrm{Hols}$ te ( 3 ) die durch Herzmittelinjektion entstehende Entzündung erprobt hat, trat zwar wiederholt, aber nicht mit Sicherheit eine lokale Reaktion auf. Eine solche zeig te sich auch dann nicht immer, wenn das Tier durch Vorbehandlung mit Natriumoxalat (Kalkentziehung) für das Auftreten von Entzündung sensibilisiert worden war. Erst am Hunde traten, wie das bereits P. Ka u f ma n n (4) berichtet, nach - selbst streng aseptisch ausgeführter - Injektion kleinster Digitoxinmengen $(0,0001 \mathrm{~g})$ entzündliche Infiltrate auf.

In der zweiten Versuchsreihe wurden $z$ ugleich mit den entzündlich wirkenden Heilmitteln Anästhetika subkutan injiziert und zur Kontrolle eine gleiche Dosis Gift dem Hunde ohne Anästhetikum gfeichzeitig an der entsprechenden Stelle der andern Körperhälfte eingespritzt. (S. Tabelle 1 .)

Diese Versuche zeigen also, daß bei Einspritzungen der genannten Heilmittel am Hunde $2-3 \mathrm{cg}$ Anästhetikum die sonst hervorgerufene Entzündung hintanhalten.

Adrenalin $(0,0001 \mathrm{~g})$ verhindert diesen Erfolg; es verzögert die Resorption so stark, daß die Anästhesie zu einem Zeitpunkt abgeklungen ist, in dem das reizende Heilmittel seine unverwünschte örtliche Wirkung noch entfalten kann. 
Tabelle 1 .

Injektion entzündtich wirkender Heilmittel am Hunde mit und ohne Zusatz von Anaesthetikum.

\begin{tabular}{|c|c|c|c|c|c|c|c|}
\hline Präparat & Zusatz & 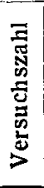 & 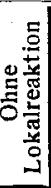 & $\begin{array}{l}\text { Mit } \\
\text { Lokalreaktion } \\
\text { und zwar: }\end{array}$ & 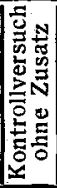 & 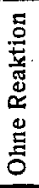 & $\stackrel{\text { Mit }}{\text { Lokalreaktion }}$ \\
\hline 0,0001 g Digitoxin 1 ) & - & 3 & ᄂ & $3 \times$ infiltrat & - & - & - \\
\hline $1 \mathrm{ccm}$ Scillaren .. & 一 & 4 & 1 & $\begin{array}{l}2 \times \text { Infittrat } \\
1 \times \text { Rötung }\end{array}$ & - & - & - \\
\hline $1 \mathrm{ccm}$ Digipurat. . & - & 4 & 1 & $3 \times$ Rötung & - & - & - \\
\hline $1 \mathrm{ccm}$ Digaten . . & $\div$ & 4 & - & $\begin{array}{l}2 \times \text { Infiltrat } \\
2 \times \text { Rötung }\end{array}$ & - & - & - \\
\hline \multirow{3}{*}{$\begin{array}{l}1 / 4 \mathrm{~cm} \text { Strophanthin } \\
1 \mathrm{ccm} \text { Cymarin .. }\end{array}$} & 一 & 6 & - & $6 \times$ Infiltrat & - & $\cdots$ & - \\
\hline & - & 3 & 1 & $2 \times$ infittrat & - & - & - \\
\hline & Summe: & 24 & 3 & 21 & - & - & - \\
\hline 0,0001 g Digitoxin & $\begin{array}{l}1 \text { ccm } 2 \% \text { ige } \\
\text { Novok. hydr.- } \\
\text { Losung }\end{array}$ & 10 & 9 & $1 \times$ Rötung & 10 & - & $10 \times$ infittrat \\
\hline $1 \mathrm{ccm}$ Scillaren & $\begin{array}{c}0,03 \text { Stovain. } \\
\text { hydr. in } 1 \mathrm{ccm}\end{array}$ & 4 & 4 & - & 4 & 1 & $3 \times$ Rötung \\
\hline $1 \mathrm{ccm}$ Digipurat & $\begin{array}{l}\text { 0,03 Novokain. } \\
\text { hydr. in } 1 \mathrm{ccm}\end{array}$ & 4 & 3 & $1 \times$ Rötung & 4 & 1 & $3 \times$ infittrat \\
\hline l'ccm .Digalen & $\begin{array}{l}\text {,02 Alyp. nitr. } \\
\text { in } 1 \mathrm{ccm}\end{array}$ & 2 & 20 & - & 2 & - & $2 \times$ Rötung \\
\hline $1 / 1, \mathrm{ccm}$ Strophanthin & $\begin{array}{l}0,03 \text { Novokain. } \\
\text { hydr. in } 1 \mathrm{ccm}\end{array}$ & 12 & 11 & $1 \times$ infiltrat $\left.^{2}\right)$ & 12 & - & $\begin{array}{c}2 \times \text { Rötung } \\
10 \times \text { infililtrat }\end{array}$ \\
\hline \multirow[t]{2}{*}{$1 \mathrm{ccm}$ Cymarin } & $\begin{array}{l}0,03 \text { Aly p. nitr. } \\
\text { in } \cdot 1 \text { ccm }\end{array}$ & 3 & 3 & -. & 1 & - & $1 \times$ Schwellung \\
\hline & & 35 & & & 33 & 2 & |31 \\
\hline
\end{tabular}

B. Klinische, Versuche ${ }^{3}$ ): Jeder Patient erhielt im allgemeinen nur eine Einspritzung. Zur Kontrolle wurde in zahlreichen Fällen dieselbe Dosis des Medikaments an der andern Körperhältte ohne Anästhetikum injiziert, mit Ausnahme jener, bei denen die schwere entzündliche Reizung mit Sicherheit feststeht. Nur von Strophanthin und Cymarin, die bisher subkutan nicht gegebent werden konnten, und bei dem in die Praxis neu eingeführten Scillaren wurde in einigen Fällen die Heilwirkung durch wiederholte Injektionen am selben Patienten erprobt.

Es seien zunächst einige Vorsichts maBregeln vorausgeschickt, die erfüllt sein müssen, damit beim Patienten die entzündliche Reaktion verhindert wird:

1. Intrakutane Quaddeln müssen sorgfältig vermieden werden, weil in ihnen die Resorption so verlangsamt ist, daß nach Abklingen der Anästhesie noch Entzündung auftritt; auch soll aus dem gleichen Grunde in ödematösem Gebiet nicht injiziert werden. Die Injektionşnadel darf vor dem Einstich mit der Injektionsflüssigkeit nicht in Berührung kommen.

2. Adrenalinzusatz schadet gleichtalls durch Resorptionsverlangsamung: die käuflichen Novokaịn-Adrentinampullen sind daher ungeeignet.

3. Injektion in die Nähe von Nervenstämmen ist zur Vermeidung von Neuralgien peinlichst zu vermeiden. Weitaus am günstigsten erwies sich in dieser Hinsicht subkutane Einspritzung im oberen, äuBern Quadranten des Gesäßes.

4. Patienten mit multipler Sklerose, Neuritiden, Hemiplegiker auf der gelähmten Seite reagieren aut die Injektion trotz des Anästhetikums oft mit Entzündung. Es scheint sich hierbei um den Ausfall eines hemmenden Einflusses der nervösen Zentren zu handeln. (Zu diesem Punkt siehe auch F. B. H of $\mathrm{m}$ a n $n(5)$, F. A. Ka u f m a n nu. M. Winkel(6).) Die Methode ist daher bei diesen Kranken nicht anzuwenden.

5. Wenn es auch überflüssig sein mag, sei der Vollständigkeit halber erwähnt, daß Novokaini zur Sterilisation nur kurz aufgekocht werden darf; es zersetzt sicl sonst unter Braunfärbung und hat geringere Wirksamkeit. gefaBt.

Die Ergebnisse dieser Versuche sind in Tabelle. Il zusammen-

Zu dieser Tabelle sei noch bemerkt: D i ga le n (Hoffmann-La Roche) reizt das menschliche Gewebe auch ohme Anästhetikum relativ wenig. Digipurat (Knoll) und Diginorgin (Norgine) werden durcli das Anästhetikum ausgefällt; ihr geringer Dispersitätsgrad bewirkt dann. Resorptionsverlangsamung, die die Anästhesie überdauern und so doch rioch. Entzündung bewirken kanit. Scillare n (Sandoz) wurde auch therapeutisch angewendet (1-2 Ampullen tgI.) bis zum Eintreten der Bradykardie usw. 1nnd zeigte auf Diurese usw. den bekannten günstigen Einfluß der Scillapräparate. Cynarin (Bayer) erwies sich auch bei subkutaner Injektion als ein wertvolles Herzmittel in jenen Fällen, in welchen man mit Digitalis nicht aus: reicht. (Jeder: 2. Tag eine Ampulle.)

Die größte AnzahlklinischerVersuche wurde mit Strophanthin (Boehringer) vorgenomment. Es ergab sich nun, daß Stro-

1) $0,1 \mathrm{ccm}$ einer Lösung von 0,03 Digitox. crystallisat. Merck auf $12 \mathrm{ccm}$ absol. Alk tnd $18 \mathrm{ccm} \mathrm{Aqu.dest.} \mathrm{-} \mathrm{2)} \mathrm{intrakutane} \mathrm{Quaddel.} \mathrm{--.} \mathrm{3)} \mathrm{Die} \mathrm{Gelegenheit} \mathrm{zur} \mathrm{Ausführung}$ der Injektionen an Patienten verdanken wir dem Entgegenkommen der Leiter der 11. Medizinischen Klinik, Prof. Dr. F. Ch vostek, der Herzstation, Prof. Dr. Ka u f ma n n, und der Klinik für Dermatologie und Syphilidologie, Prof. Dr. Rieh1.
Tabelle t1.

Injektion entzündlich wirkender Heilmittel mit Zusatz von Anaesthetikum an Patienten

\begin{tabular}{|c|c|c|c|c|c|c|c|}
\hline Präparat & Zusatz & 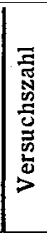 & 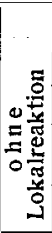 & $\begin{array}{c}\text { Davon } \\
\text { m it } \\
\text { Lokalreaktion }\end{array}$ & 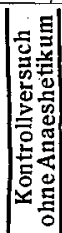 & 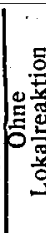 & $\begin{array}{c}\text { Mit } \\
\text { Lokalreaktion }\end{array}$ \\
\hline $1 \mathrm{ccm}$ Scillaren & $\begin{array}{l}1 \operatorname{ccm} 2 \% \text { ige } \\
\text { Novokainlös. } 1)\end{array}$ & 2 & - & $1 \times$ Rötung & 2 & - & $2 \times$ Schweltung \\
\hline $1 \mathrm{ccm}$ Scillaren & $\begin{array}{l}1 \operatorname{ccm} 5 \% \text { ige } \\
\text { Novokainlös. }\end{array}$ & 44 & 38 & $\begin{array}{l}4 \times \text { Schwellung } \\
2 \times \text { Rötung }^{2} \text { ) }\end{array}$ & 22 & 4 & 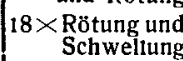 \\
\hline $1 \mathrm{ccm}$ Digalen & $\begin{array}{l}1 \mathrm{ccm} 5 \% \text { ige } \\
\text { Novokainlos. }\end{array}$ & 3 & 3 & - & $t$ & - & $\mathrm{t} \times$ Rötung \\
\hline $1 \mathrm{ccm}$ Digipurat & $\begin{array}{l}1 \mathrm{ccm} 5 \% \text { ige } \\
\text { Novokainlös. }\end{array}$ & 17 & 11 & $\begin{array}{l}4 \times \text { Rötung } \\
\left.2 \times \text { Infintrat }^{3}\right)\end{array}$ & 10 & 1 & $\begin{array}{l}9 \times \text { Rötung und } \\
\text { Schwellug }\end{array}$ \\
\hline $1 \mathrm{ccm}$ Diginorgin & $\begin{array}{l}1 \mathrm{ccm}^{*} 5 \% \text { ige } \\
\text { Novokainlos. }\end{array}$ & 4 & 1 & $\begin{array}{l}2 \times \text { Rötung } \\
1 \times \text { Infiltrat }\end{array}$ & 1 & - & 1 Infiltrat \\
\hline $2, \mathrm{ccm}$ Cymarin & $\begin{array}{l}1 \mathrm{ccm} 5 \% \text { ige } \\
\text { Alypinlösung }{ }^{4} \text { ) }\end{array}$ & 5 & 5 & - & 1 & - & $\begin{array}{c}1 \text { grobes } \ln \text { - } \\
\text { filtrat }\end{array}$ \\
\hline $1 / 4-1 \mathrm{ccm}$ Stroph. & $\begin{array}{l}1 \text { ccm } 5 \% \text { ige } \\
\text { Novokainiós. }\end{array}$ & 63 & 55 & $\begin{array}{l}\left.5 \times \text { Infiltrat }^{5}\right) \\
3 \times \text { Rötung } \\
\end{array}$ & - & - & - \\
\hline & Summe & $\mid 138$ & 113 & $\left.25^{6}\right)$ & 37 & 5 & 32 \\
\hline
\end{tabular}

phanthin mit Novokain. hoydrochlor. oder Alypin. nitric. unter den oben angegebenen Vorsichtsmabregeln ohne jede Lokalreaktion subkutan gegeben werden kann. Aus der Zahl der Beobachtungen der klinischen Wirkung der subkutanen Strophanthininjektion sei der Kürze halber nur folgender Einzelfall mitgeteilt:

Karl K., 50 Jahre, Mesaortitis luica und Aorteninsuffizienz, ausgedehnte Oedeme, Dyspnoe, Puls um 120, unregelmäßig, schon vorher wochenlang erfolglos behandelt. Das folgende Diagramm zeigt den Einfluß der 10 subkutanen Strophanthininjektionen auff die Diurese:

Die Injektionen wurden vom 31. III. bis 18. IV. 1922 jeden zweiten Tag gegeben, zuerst $1 / 4$, dann $1 / 2 \mathrm{mg}$, dann $1 \mathrm{mg}$ Strophanthin; vor kürzeren Zwischenpausen und rascherent Ansteigen mit der Dosis is zu warnen. Am Ende der 18 tägigen Kur waren die Oedeme vollstält ig g e schwunde 11 , der Puls 72, nur noch zeitweise unregelmäBig. Dic subjektiven Beschwerden: Schmerzen, Schlaflosigkeit und Husten waren beseitigt.

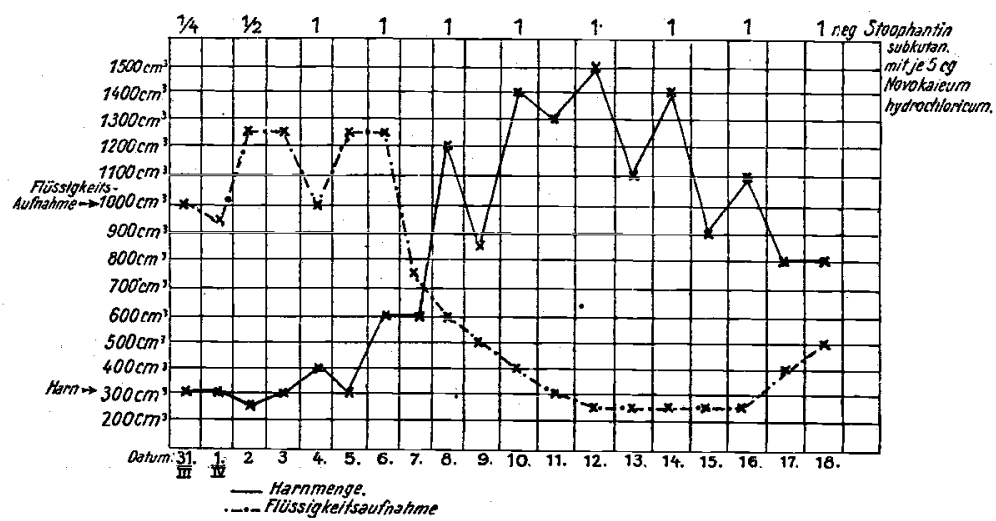

Zusammenfassung. 1. Digalen, Digipurat, Scillaren, Cymarin und Strophanthin lassen sich nach Zusatz von Anästhetika reizlos subkutan injizieren.

2. Dic Indikationsstellung für die Therapie mit Cymarin und Strophanthin wird bedeutend erweitert, ihre Anwendung erleichtert.

1. M. m. W. 1906. - 2. Arch. f. exper. Path. u. Pharm. 1910. - 3. Arch. f. exper. Path. u. Pharm. 1913. - 4. Arch. f. exper. Path. u. Pharm. 1887. - 5. Zschr. f. Biol. 1920. 6. KI. W. 1922 . 